\title{
Research on Rainstorm Risk Evaluation System Construction in Sichuan Province
}

\author{
Ding Chen ${ }^{1}$, Tiangui Xiao ${ }^{1}$, Fengrong Jing ${ }^{1}$, Jianglin Fan², Weiwei Gan ${ }^{3}$, Li Wang ${ }^{2}$, \\ Zhaoyu Zhan ${ }^{3}$, Yujie Fang ${ }^{4}$, Chao Wang ${ }^{1}$, Yawen Zhu ${ }^{1}$ \\ ${ }^{1}$ Chengdu University Of Information Technology, Chengdu 610225, China \\ ${ }^{2}$ Sichuan Meteorological Observatory, Chengdu 610071, China \\ ${ }^{3}$ Sichuan Climate Center, Chengdu 610071, China \\ ${ }^{4}$ Weather bureau of Pixian, Chengdu city, Pixian 611700, China

\section{四川省暴雨灾害风险评估系统构建研究} \\ 陈丁 ${ }^{1}$, 肖天贵 ${ }^{1}$, 敬枫蓉 ${ }^{1}$, 范江琳 ${ }^{2}$, 甘薇薇 ${ }^{3}$, 汪丽 ${ }^{2}$, 詹兆渝 ${ }^{3}$, 房玉洁 ${ }^{4}$, 王超 ${ }^{1}$, \\ 朱雅文 ${ }^{1}$ \\ ${ }^{1}$ 成都信息工程大学, 成都 610225 , 中国 \\ 2 四川省气象台, 成都 610071 , 中国 \\ ${ }^{3}$ 四川省气候中心, 成都 610071 , 中国 \\ ${ }^{4}$ 成都市郫县气象局, 郫县 611700 , 中国
}

\begin{abstract}
This paper based on the Sichuan daily precipitation data, geographic information and historical data such as disaster,then using industry proven lightweight Java EE frameworks to building the business of system and the evaluation model implemented by Fortran language, Finally completion of the rainstorm process by level assessment. All data within the system to complete your interactions and analysis, automatic generation of assessment reports, improves the efficiency and level of rainstrom disaster protection.
\end{abstract}

Keywords: Sichuan area, Rainstrom Disaster, Risk Evaluation, System Construction

\section{摘要}

本文以四川省逐日降水数据、地理信息及历史 灾情等数据为基础, 利用业界公认的轻量级 Java EE 框架及 Fortran 语言构建业务系统
和评估模型, 最终完成对暴雨过程的等级评估。 所有数据均在系统内部完成分 析计算, 自动 生成评估报告, 提高了暴雨灾害防护技术服务 的工作效率和水平。

关键词：四川地区, 暴雨灾害, 风险评估, 系统构建

1. 引言

极端气象灾害如暴雨洪涝、干旱、暴雪、 高温热浪、低温冷害等日益频繁, 造成了国 民经济和人民生命财产的巨大损失, 引起各 级政府和百姓的广泛重视。在这些重大的气 象灾害事件中, 强降水致灾的影响极为突出, 强降水所导致的灾害在我国致灾气象事件 中所占比例高达 $40 \%$, 也是四川省危害最重 的气象灾害。因而, 基于现代技术对区域性 暴雨灾害的预警及风险评估的业务化是气 象业务现代化中的重要任务。本项工作基于 暴雨致灾的孕灾环境、致灾因子、承载体相 互作用的气象灾害的形成机制, 建立了暴雨 
Risk Analysis and Crisis Response in Big Data Era (RAC-16)

等级评估模型、层次分析评估模型、模糊评 估模型、可拓理论评估模型, 结合 GIS 技术 对空间信息的管理能力与 DSS 技术强大的 辅助决策功能, 构建和开发了基于 GIS 技术 的四川省暴雨灾害的气象灾害监测、预报预 警和风险评估系统, 实现了对暴雨过程的预 警和风险评估的信息化管理与维护的自动 化、信息传输与发布的网络化、信息分析处 理的模型化、信息服务与决策的可视化, 推 进了四川地区气象现代化的业务工作。

\section{2. 体系设计}

首先讨论系统数据库和模型库的设计。 在对暴雨预警和风险评估及气象要素大数 据特征进行深入分析后, 给出了风险评估系 统的模型及体系结构, 并进行了系统模块划 分。利用四川省历年逐日、逐小时历史降水 资料和社会经济、灾情调查等基础资料, 构 建四川省强降水雨情和灾情查询专题数据 库。根据统计理论、层次分析、模糊理论、 可拓理论等方法对四川地区强降水致灾特 征的研究, 建立一套合理的降水致灾评估模 型, 形成灾前、灾中、灾后快速评估并及时 准确地发布灾前、灾中、灾后灾害评估产品。 利用 GIS 系统的空间分析和管理能力, 充分
挖掘各类数据间的联系, 全面提升系统的应 用水平和各类数据的使用效率, 形成可视化 的灾前、灾中、灾后评估产品。系统还提供 直观、形象、方便、图形化的统计分析工具 和显示手段, 使系统具备强大的可视化分析 能力。

\section{3. 系统结构}

按照系统需求并结合对业务的整体分 析, 整个系统分为以下 5 个层次: 表现层、 业务系统层、基础服务层、数据层、系统平 台。表现层, 是系统和用户直接进行交互, 可以基于 PC 端、手机端和 LED 大屏进行展 示和使用; 业务系统层是整个系统能够实现 的功能情况, 包括气象信息综合查询、气象 实况监测、气象灾害评估、气象灾情预报、 灾情预警发布、后台管理等六大子系统; 数 据层作为系统的数据核心, 主要包括地理信 息基础数据库、专题数据库、业务数据库, 其中业务数据库分为: 气象监测、风险评估、 预警信息; 专题数据库分为强降水专题数据 库、强降水历史灾情专题数据库; 系统平台 作为系统的开发环境和运行环境, 主要包括 了 WebGIS 平台、Java EE 框架、操作系统和 数据库管理系统, 以及配套的硬件环境。暴

\section{四川暴雨灾害风险评估系统总体架构}

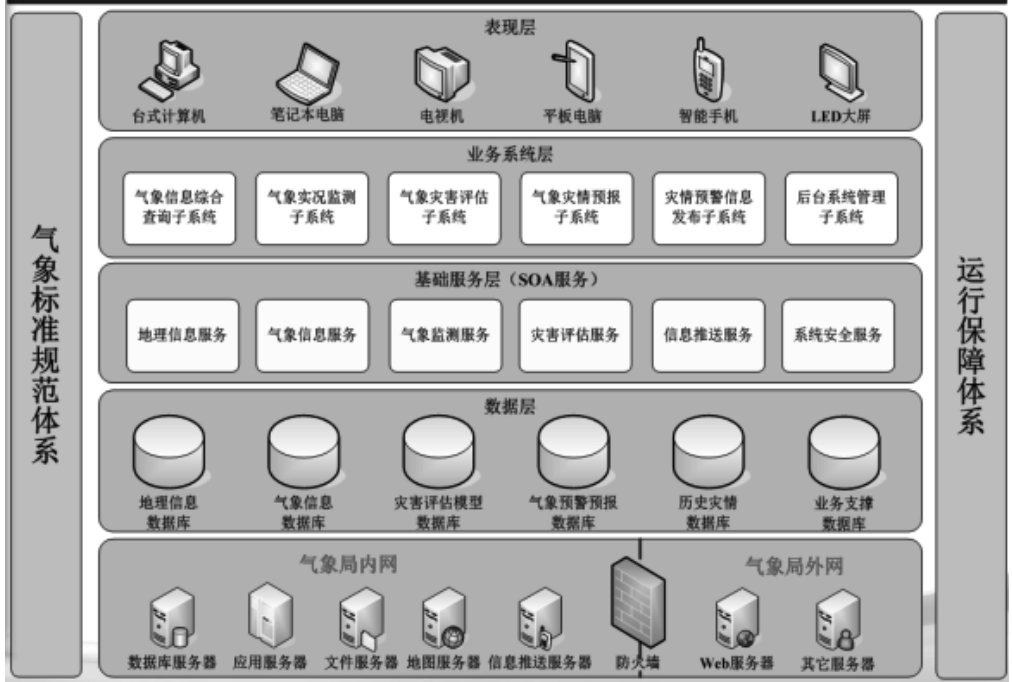

图 1: 系统总体架构 


\section{Risk Analysis and Crisis Response in Big Data Era (RAC-16)}

雨灾害风险评估系统的总体架构如图 1所示。 系统信息处理的流程如图 2 所示。
统评估模型多是使用 Fortran 进行编写, 为 实现业务系统的可扩展性, 我们提出了一种

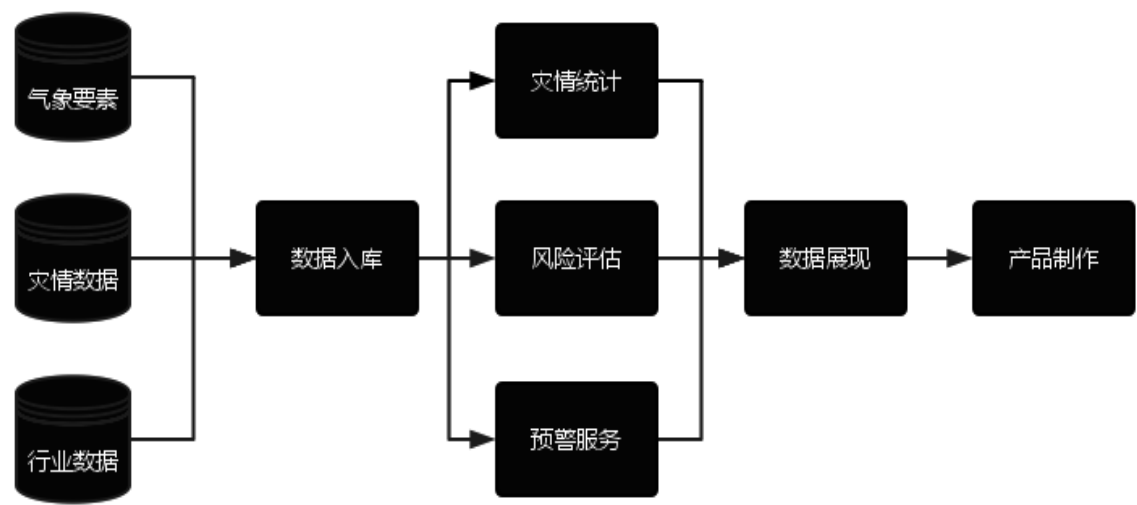

图 3: 系统数据处理流程

本系统实现采用轻量级的 Java EE 三层 架构体系, 应用 SOA/MVC 的设计模式, 运用 了大量先进的诸如 Ajax 等 Web2.0 的技术, 实现了 “高内聚, 低耦合” 的设计思想, 增 强了系统的柔性和开放性。展现层使用 JQuery、Echart 等成熟框架来丰富表现形式, 业务层采用稳定、快速的 Spring 框架来实 现系统的核心业务, 数据层采用最新的 JPA 标准来实现实体/关系映射。系统的软件技 术体系结构如图 3 所示。

根据系统总统结构的设计, 细化系统功 能模块如图 4。

\section{4. 系统特点}

(1) Java 和 Fortran 混合编程。

在实现业务系统的过程中, 我们发现系
基于 Java 和 Fortran 混合编程的解决方案。 由 Java 负责系统的 Web 展现和控制, Fortran 实现评估模型算法, 充分发挥 Java 在网络编程的优势和Fortran 强大的计算能 力。这样不仅可减少重复性开发, 还提高了 系统的扩展性, 一旦评估模型变化只需要替 换 Fortran 文件, 业务系统不需要做任何变 更。

(2) 自动化评估报告生成。

该系统将评估报告作为一个核心的基础服 务支撑模块, 在对暴雨过程进行预估和评估 后, 本系统将自动生成一份评估报告, 减轻 重复性的人力劳动工作。评估人员也可以将 典型的评估报告上传至范例库, 方便其它地、 市、州的评估工作人员在处理相同或类似问 题时吸取经验、获取知识, 再针对新

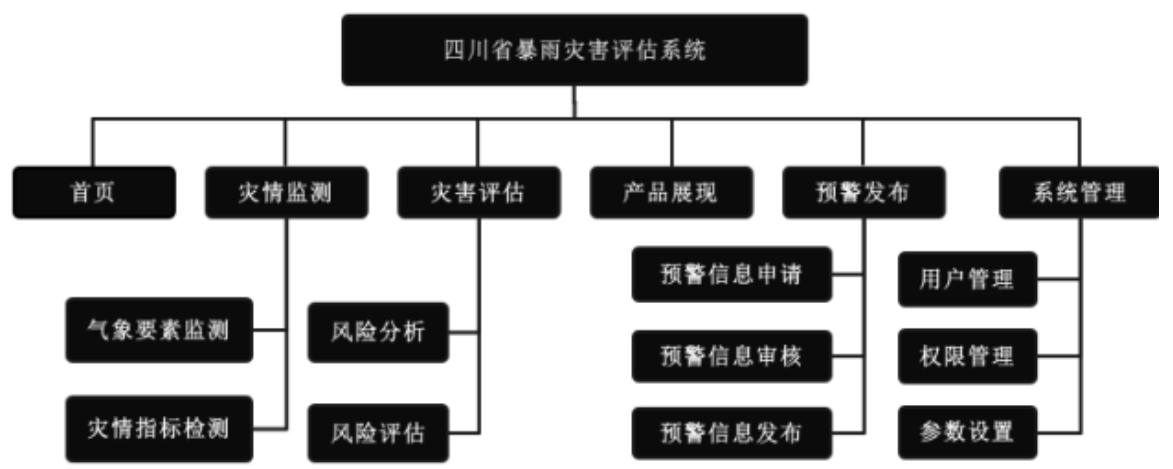

图 2: 系统功能模块 
Risk Analysis and Crisis Response in Big Data Era (RAC-16)

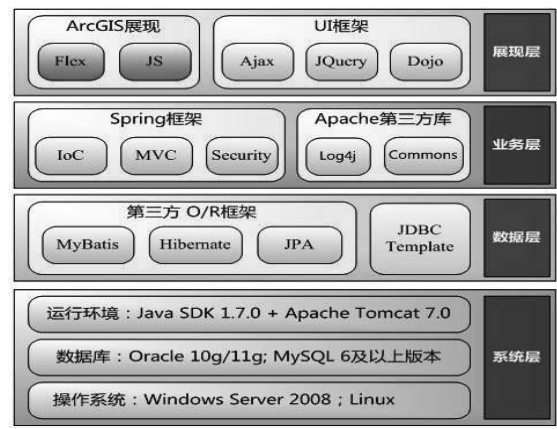

图 4: 系统软件技术体系

旧情况的差异做出相应的调整, 从而形成解 决方案。

(3) WebGIS 展现。

本系统利用 GIS 的空间分析能力, 将每 天的降水数据, 经过插值计算、掩膜提取、 栅格渲染和等值线分析后, 形成雨量分布图,
供预估人员直观了解某辖区的降水变化情 况。

\section{5. 系统运行初步结果}

利用1961-2013年四川省156个国家级 气象观测站的逐日降水资料, 选取 $08 \mathrm{~h}$-次 日08h降水量建立暴雨过程评估模型的统计 分析数据集, 按此标准, 获得了四川省 1961-2013年共856次暴雨过程, 系统的等级 评估模型的概括率达到 $85 \%$ 。

系统开发安装后试运行, 在 2016 年 1 $\sim 5$ 月初试运行期间, 灾前预评估 3 次暴雨过程 和灾中、灾后暴雨风险评估, 暴雨过程等级、 持续时间、暴雨强度等基本符合实际业务要 求（图 5)。进一步对气象灾害预警和风险 评估模型完善后，区域性暴雨预警和风险评 估效果会有显著提高。

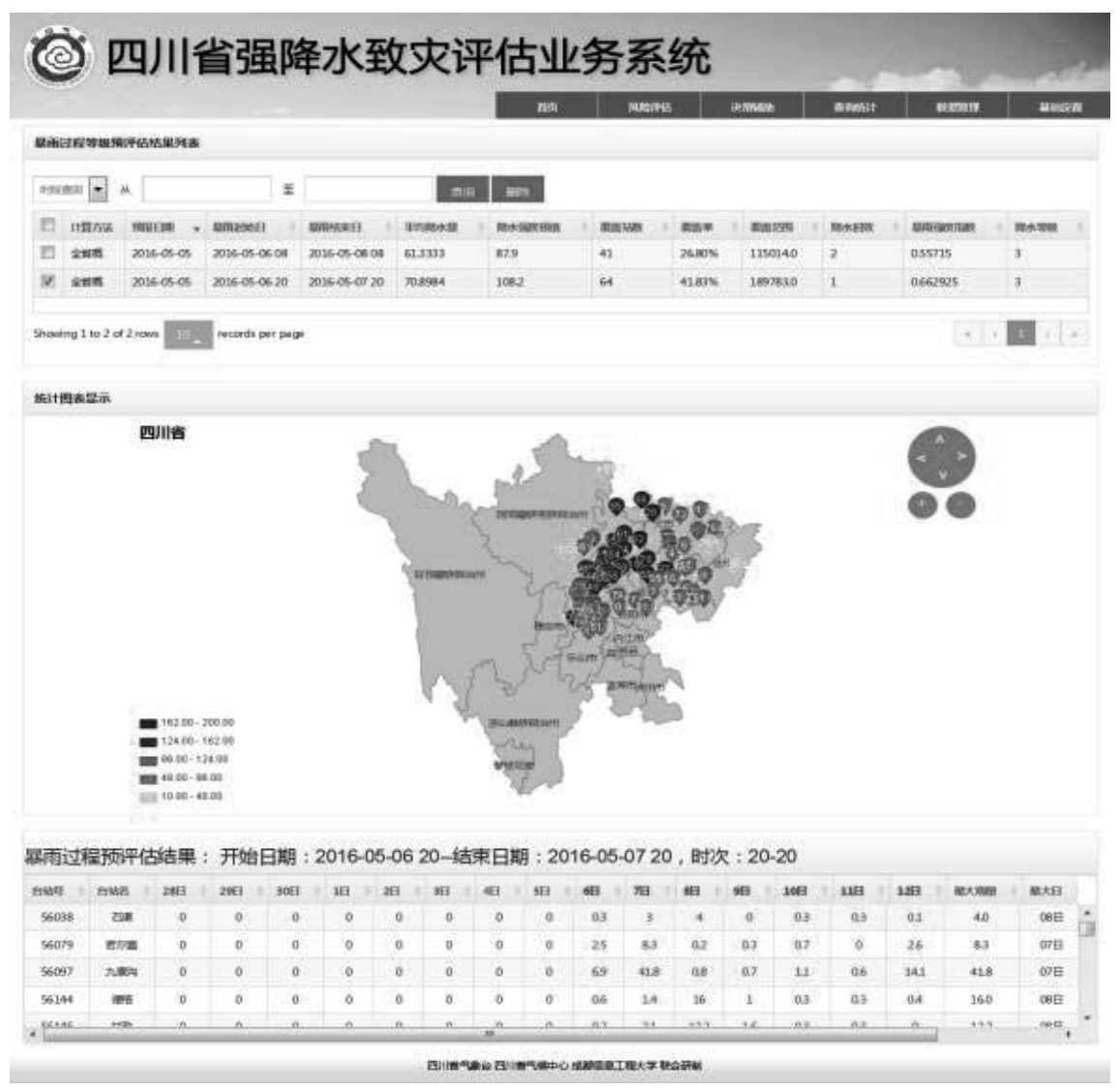

图 5: 暴雨过程预评估结果 
Risk Analysis and Crisis Response in Big Data Era (RAC-16)

\section{Acknowledgements}

This study was supported by Southwest Regional Meteorological Center of China Meteorological Administration major research projects (Southwest Region014-5)、 National Natural Science Foundation of China Fund Project (91337215), and the National Key Basic Research Program (2013CB733206), Sichuan Province, applied basic research projects (2013JY0063)。

\section{致谢}

本研究得到了中国气象局西南区域气象 中心重大科研项目（西南区域 014-5）、国家 自然科学基金重点基金项目（91337215）、国 家重点基础研究发展计划 (2013CB733206)、 四川省应用基础研究计划项目（2013JY0063） 的资助。

\section{参考文献}

[1] C.Y. Song, R. Zhang, A.X. Zhou, M. Hong. Water security risk assessment of the yellow river basin based on constrained-random weight and cloud model. Journal of Risk Analysis and Crisis Response, 2013, 3(4): 201-209

[2]丁一汇, 张建云. 暴雨洪涝. 北京: 气象出版 社,2009.

[3]A. Lerner-Lam. Assessing global exposure to natural hazards: Progress and future trends. Environmental Hazards, 2007, 7:10-19

[4]陈艳秋,袁子鹏.辽宁暴雨事件影响的预评估 和灾后速评估.气象科学, 2007, 27(6): 626-632.

[5]邹燕,叶殿秀,林毅,等.福建区域性暴雨过程 综合强度定量化评估方法. 应用气象学报, 2014,235(3):360-364. 